\title{
Huang-Pu-Tong-Qiao Formula Ameliorates Tau Phosphorylation by Inhibiting the CaM-CaMKIV Pathway
}

\author{
Shu Ye $\mathbb{D}^{1},{ }^{1,2}$ Biao Cai $\mathbb{D}^{1,2,3}$ Peng Zhou ${ }^{1},{ }^{1,2,3}$ Guoquan Wang ${ }^{1},{ }^{1}$ Huawu Gao, ${ }^{1,2}$ \\ Rupeng Hua $\mathbb{D}^{1},{ }^{1}$ Liangzhen You, ${ }^{1}$ Yongchuan Yao ${ }^{\mathbb{D}},{ }^{4}$ Yan Wang $\mathbb{D},{ }^{1,2}$ \\ and Guoming Shen $\mathbb{D}^{1,2}$

\footnotetext{
${ }^{1}$ School of Integrated Chinese and Western Medicine, Anhui University of Chinese Medicine, Hefei 230012, Anhui, China

${ }^{2}$ Institute of Integrated Chinese and Western Medicine, Anhui Academy of Chinese Medicine, Hefei 230012, Anhui, China
} \\ ${ }^{3}$ Anhui Province Key Laboratory of Chinese Medicinal Formula, Hefei 230012, Anhui, China \\ ${ }^{4}$ The First Affiliated Hospital of Anhui University of Traditional Chinese Medicine, Hefei 230031, Anhui, China
}

Correspondence should be addressed to Yan Wang; abudao2002@163.com and Guoming Shen; shengm_66@163.com

Received 21 January 2020; Accepted 9 March 2020; Published 12 May 2020

Academic Editor: Tadaaki Satou

Copyright $\odot 2020$ Shu Ye et al. This is an open access article distributed under the Creative Commons Attribution License, which permits unrestricted use, distribution, and reproduction in any medium, provided the original work is properly cited.

\begin{abstract}
Alzheimer's disease (AD) is a complex neurodegenerative disease. It is a chronic, lethal disease in which brain function is severely impaired and neuronal damage is irreversible. Huang-Pu-Tong-Qiao (HPTQ), a formula from traditional Chinese medicine, has been used in the clinical treatment of $\mathrm{AD}$ for many years, with remarkable effects. However, the neuroprotective mechanisms of HPTQ in AD have not yet been investigated. In the present study, we used $\mathrm{AD}$ models in vivo and in vitro, to investigate both the neuroprotective effect of HPTQ water extracts (HPTQ-W) and the potential mechanisms of this action. For the in vivo study, after HPTQ intervention, the Morris water maze test was used to examine learning and memory in rats. Transmission electron microscopy and immunofluorescence methods were then used to investigate neuronal damage. For the in vitro experiments, rat primary hippocampal neurons were cultured and cell viability was examined by 3-(4,5-dimethylthiazol-2-yl)-2,5-diphenyltetrazolium bromide. Additionally, mRNA levels of CaM, CaMKK, CaMKIV, and tau were examined using qRT-PCR, and protein expression of CaM, CaMKK, p-CaMKIV, and p-tau were examined using western blot. In vivo, we revealed that HPTQ significantly improved learning and memory deficits and attenuated neuronal damage in the AD rat model. Furthermore, in vitro results showed that HPTQ significantly increased cell viability in the AD cell model. We also demonstrated that HPTQ significantly decreased the mRNA levels of CaM, CaMKK, CaMKIV, and tau and significantly decreased the protein expressions of CaM, CaMKK, p-CaMKIV, and p-tau. In conclusion, our results indicated that HPTQ improved cognition and ameliorated neuronal damage in AD models and implicated a reduction in tau phosphorylation caused by inhibition of the CaM-CaMKIV pathway as a possible mechanism.
\end{abstract}

\section{Introduction}

Alzheimer's disease (AD) is an age-related neurodegenerative disease [1] that is characterized by cognitive deficits, memory loss, language impairments, and mental and behavioral alterations $[2,3]$. Extracellular $A \beta$ deposition forms senile plaques, and intracellular hyperphosphorylated tau produces neurofibrillary tangles (NFTs); these are the two hallmark histopathological lesions of AD. The World Alzheimer's Report of 2018 reported that 50 million people worldwide were living with dementia in 2018, and this number is expected to more than triple to 152 million by 2050. The cost of dementia is enormous, having reached $\$ 1$ trillion by 2018, and is still increasing [4]. However, the mechanisms of $\mathrm{AD}$ are complex and may include the cholinergic hypothesis, the amyloid-beta $(\mathrm{A} \beta)$ hypothesis, the tau hypothesis, the neurotoxicity hypothesis, and the oxidative stress hypothesis, among others [5].

Tau is a microtubule-associated protein that plays an important role in the synthesis and stabilization of the neuronal cytoskeleton to maintain cell integrity [6]. Abnormal hyperphosphorylation of tau ( $\mathrm{p}$-tau) decreases the 
binding affinity of microtubules, resulting in NFTs. These changes have been associated with multiple neurodegenerative disorders and are closely related to AD severity $[7,8]$. Tau can be phosphorylated in 85 residues [9], by kinases such as glycogen synthase kinase 3 (GSK-3), mitogen-activated protein kinase (MAPK), and $\mathrm{Ca}^{2+}$-calmodulin-dependent protein kinase II (CaMKII) [10]. These kinases are considered to be targets for anti-p-tau therapies. Studies have shown that Tideglusib, a GSK-3 inhibitor, improves cognition in double transgenic mice expressing human tau and amyloid precursor protein (APP) by reducing tau phosphorylation, amyloid deposition, and neuronal loss [11].

However, the treatment of AD has historically been very difficult and remains challenging in the present. There are currently four FDA-approved medications for improving AD symptoms, including cholinesterase inhibitors, such as donepezil, and the uncompetitive NMDA receptor modulator memantine. In addition, some novel approaches (including those involving BChE inhibition, antiamyloid agents, tau protein and related enzymes, and antioxidants) can alleviate patients' conditions, but do not modify $\mathrm{AD}$ progression. So far, amyloid-based therapies have fallen into the bottleneck in improving the course of $\mathrm{AD}$, and tauoriented therapies are still in the early stages of development [12].

Huang-Pu-Tong-Qiao (HPTQ) is a hospital formula from the First Affiliated Hospital of Anhui University of Traditional Chinese Medicine and is mainly composed of Shi Chang-pu, Yi-zhi, He Shou-wu, Chuang-xiong, Da-huang, and Ren-sheng. This formula has been used to treat dementia for more than 10 years and has a remarkable curative effect; however, the mechanism of this effect is unclear. We hypothesized that HPTQ might reduce tau hyperphosphorylation, thus achieving its effective treatment of AD. To elucidate the mechanism of HPTQ in the treatment of $\mathrm{AD}$, we used intraperitoneal injections of $\mathrm{D}$-galactose combined with intraventricular injections of $\mathrm{A} \beta_{25-35}$ to establish an $\mathrm{AD}$ animal model and used $\mathrm{A} \beta_{25-35}$ to induce an $\mathrm{AD}$ cell model in primary hippocampal neurons. Histopathological changes and cellular biomarkers were examined.

\section{Materials and Methods}

2.1. Materials. Stilbene glycoside, ferulic acid, ginsenoside Rg1, aloe-emodin, $\beta$-asarone, emodin, and chrysophanol were purchased from China National Institutes for Food and Drug Control (Beijing, China). Nimodipine was purchased from Bayer Medical and Health Co., Ltd. (Beijing, China). Neurobasal-A media (NM) and B27 were purchased from Gibco Industries Inc. (Auckland, New Zealand). Poly-Dlysine (PDL) and $\mathrm{A} \beta_{25-35}$ were purchased from SigmaAldrich (St. Louis, MO, USA). KN-62 was purchased from Selleck Chemicals (Houston, TX, USA).

2.2. A $\beta$ Aggregation. $\mathrm{A} \beta_{25-35}$ was added to sterile doubledistilled water at a concentration of $2 \mathrm{mg} / \mathrm{mL}$ or $1 \mathrm{mg} / \mathrm{mL}$ as the mother liquor for the in vivo and in vitro assays, respectively. The mother liquor was incubated at $37^{\circ} \mathrm{C}$ for 4 days and then stored at $-20^{\circ} \mathrm{C}$.

\subsection{Preparation and Ultra-Performance Liquid Chromatog-} raphy (UPLC) Analysis of HPTQ. The HPTQ solution was prepared fresh every 3 days by boiling the following six components in water: Shi Chang-pu $(20 \mathrm{~g})$, Yi-zhi $(20 \mathrm{~g})$, He Shou-wu (20 g), Chuang-xiong (20 g), Da-huang (6 g), and Ren-sheng (10 g). Tenfold water was added and refluxed for $2 \mathrm{~h}$ for the first time, and for the second time, 8 fold water was added and refluxed for $1.5 \mathrm{~h}$. The solution was then collected, concentrated to $50 \mathrm{mg} / \mathrm{mL}$, and stored at $4^{\circ} \mathrm{C}$.

Stilbene glycoside, ferulic acid, ginsenoside Rg1, aloeemodin, $\beta$-asarone, emodin, and chrysophanol levels in HPTQ were measured using UPLC. The Acquity BEH C18 $(2.1 \mathrm{~mm} \times 100 \mathrm{~mm}, 1.7 \mu \mathrm{m}$; Waters, Milford, MA, USA) analytical column was coupled with a column filter, and the temperature was set at $30^{\circ} \mathrm{C}$. The gradient elution with two mobile phase systems consisting of $0.05 \%(\mathrm{v} / \mathrm{v})$ phosphoric acid in distilled water (A) and acetonitrile (B) was performed as follows: $5 \%-25 \%$ B for $0-2 \mathrm{~min}, 25 \%-$ $67 \% \mathrm{~B}$ for $2-8 \mathrm{~min}, 67 \%-82 \% \mathrm{~B}$ for $8-10 \mathrm{~min}$, and $82 \%-$ $5 \% \mathrm{~B}$ for $10-12 \mathrm{~min}$, with a reequilibrium time of $2 \mathrm{~min}$. The flow rate and injection volume were $0.2 \mathrm{~mL} / \mathrm{min}$ and $1 \mu \mathrm{L}$, respectively. The (photodiode array, PDA) detector wavelength was monitored at $210 \mathrm{~nm}$.

\subsection{Neuroprotective Effect of HPTQ on AD In Vivo}

2.4.1. Animals and Treatments. A total of 40 male Sprague Dawley rats (250-300 g, 8-10 months old) were sourced from the Animal Experiment Center, Anhui Medical University, China (Certificate no. SXCK (Wan) 2011-002). The rats were fed adaptively for a week under a light/dark cycle of $12 \mathrm{~h} / 12 \mathrm{~h}$ and kept at a temperature of $25^{\circ} \mathrm{C}-30^{\circ} \mathrm{C}$.

After excluding animals with outlying results in the Morris water maze test, the rats were randomly divided into four groups: the normal control group (CTRL), the model group (MODEL), the HPTQ group $(1.41 \mathrm{~g} / \mathrm{kg}$, corresponding to three times the clinical equivalent according to the volume surface area folding algorithm), and the nimodipine group (NMDP; $20 \mathrm{mg} / \mathrm{kg}$, corresponding to three times the clinical equivalent according to the volume surface area folding algorithm). Except for the CTRL group, rats in the other groups were intraperitoneally injected with $100 \mathrm{mg} / \mathrm{kg} \mathrm{D}$-galactose once daily for 42 days. When they had undergone 21 days of D-galactose treatment, the rats were fastened to a brain stereotaxic instrument (Stoelting Co., Wood Dale, IL, USA) and injected using a microsyringe (Anting Scientific Instrument, Shanghai, China) with $5 \mu \mathrm{L}(10 \mu \mathrm{g}) \mathrm{A} \beta_{25-35}$ into the bilateral hippocampus. The injection point was located at $4.4 \mathrm{~mm}$ posterior to and $2.2 \mathrm{~mm}$ from the anterior fontanelle as the origin point, and the needle was placed $3.0 \mathrm{~mm}$ from the rat hippocampus [13]. The CTRL 
group was injected with an equal volume of saline. After treatment with D-galactose for 14 days, the HPTQ group and NMDP group rats were fed by gavage according to body mass.

2.4.2. Morris Water Maze Test. After intracerebral injection of $\mathrm{A} \beta_{25-35}$ for 7 days in rats, the classical Morris water maze method was used [14]. The experiment lasted for 6 days. For the first 5 days, the navigation experiment was performed. Rats were placed into the water from one of four quadrants, facing the wall of the pool, and the time taken to find the platform within $90 \mathrm{~s}$ was recorded. If the rats did not find the platform within $90 \mathrm{~s}$, they were placed on the platform and left there for $20 \mathrm{~s}$. On day 5, no guidance was given, and the time taken to find the platform was recorded as the escape latency. On day 6, the platform was removed and the same parameters were set as for the navigation experiments. The number of times that the rats crossed the area where the platform had been located was recorded.

2.4.3. Animal Sample Preparation. After the Morris water maze test, rats were sacrificed by anesthesia with an intraperitoneal injection of $1 \%$ sodium pentobarbital (40 mg/ $\mathrm{kg}$ ). Brain tissue was quickly removed and placed on saline ice. Some hippocampal tissue was cut to $1 \mathrm{~mm}^{3}$ size and stored in glutaraldehyde, to be used in transmission electron microscopy (TEM) experiments, and the remaining hippocampal tissue was isolated from both sides of the brain and stored at $-80^{\circ} \mathrm{C}$ for western blot and $\mathrm{qRT}$ PCR experiments.

2.4.4. Determination of the Fluorescent Expression of p-Tau. Brain tissue was fixed in $4 \%$ paraformaldehyde, then dehydrated, embedded, sectioned, and placed into $\mathrm{pH} 8.8$ for repair and sealing. Tissue was then incubated overnight at $4^{\circ} \mathrm{C}$ in primary rabbit anti-rat p-tau antibody. The following day, the brain tissue was incubated in fluorescent secondary FITC-rabbit anti-goat IgG antibody at room temperature for $2 \mathrm{~h}$, washed three times in phosphate-buffered solution (PBS), and incubated in DAPI for $10 \mathrm{~min}$. After washing the slides, an antifluorescent quencher was used to coverslip the tissue, and images were collected using an inverted fluorescence microscope.

\subsection{Effect of HPTQ on AD In Vitro}

2.5.1. Cell Culture and Intervention. The methods of culturing primary hippocampal neurons were performed according to Korkotian et al. $[15,16]$. Neonatal pups were sacrificed after immersion in $75 \%$ alcohol for 20 s. Hippocampi were dissected out and cut into $1 \mathrm{~mm}^{3}$ pieces after separating the blood vessels and meninges. The tissue was digested with $0.125 \%$ trypsin for $20 \mathrm{~min}$ at $37^{\circ} \mathrm{C}$, before DMEM/F12 medium with 10\% fetal bovine serum
(FBS) was added to terminate the digestion. The cells were separated and collected by centrifuging the cell suspension at $1500 \mathrm{rpm}$ for $5 \mathrm{~min}$. Next, the suspension was swirled gently and filtered through a 200-mesh sieve. Isolated primary hippocampal neurons were cultured in DMEM/F12 nutrient solution containing 20\% FBS. Subsequently, the cells were seeded onto poly-D-lysinecoated plates at a density of $1 \times 10^{6} / \mathrm{mL}$ and maintained at $37^{\circ} \mathrm{C}$ in an incubator with $5 \% \mathrm{CO}_{2}$. The medium was replaced with NM supplemented with 2\% B27 and $0.5 \mathrm{mM} / \mathrm{L}$ L-glutamine, and half of the medium was changed every 3 days.

Based on previous experimental results from our research group, the neurons were assigned to the following treatment groups after being cultured for 7 days: the control group (CTRL), model group (MODEL), KN-62 group (KN62), 15\% HPTQ-medicated serum group (HPTQ), 15\% HPTQ-medicated serum + KN-62 group (KN62 + HPTQ), and nimodipine group (NMDP). Cells in the MODEL group were incubated with $30 \mu \mathrm{M} \mathrm{A} \beta_{25-35}$ for $24 \mathrm{~h}$ to establish the AD cell model. Cells in the KN-62 group were incubated with $10 \mu \mathrm{M}$ of the KN-62 inhibitor for $3 \mathrm{~h}$ before the AD cell model was established. Cells in the HPTQ and NMDP groups were given 15\% HPTQmedicated serum and $7 \mu \mathrm{M}$ nimodipine solution, respectively, for $24 \mathrm{~h}$ after the establishment of the AD cell model. Cells in the KN62 + HPTQ group were given 15\% HPTQmedicated serum for $24 \mathrm{~h}$ after being preadministrated $10 \mu \mathrm{M}$ KN-62 and the establishment of the AD cell model. Groups are shown in Figure 1.

2.5.2. Cell Viability. After the cells were treated and cultured at $37^{\circ} \mathrm{C}$ in a $5 \% \mathrm{CO}_{2}$ incubator, the supernatant was removed and $20 \mu \mathrm{L}$ of $5 \mathrm{mg} / \mathrm{mL}$ MTT was added to each well and incubated for $4 \mathrm{~h}$. The supernatant was removed and $150 \mu \mathrm{L}$ DMSO was added to each well. The optical density at $490 \mathrm{~nm}$ was measured using a microplate reader $(318 \mathrm{C}+$, Shanghai Peiou Co., Ltd., Shanghai, China).

\subsection{Mechanism of HPTQ on AD In Vitro and In Vivo}

2.6.1. Quantitative Real-Time PCR ( $q R T-P C R)$. The total RNA of hippocampal tissue or treated cells was extracted according to the instructions using a TRIzol kit (Invitrogen, Carlsbad, CA, USA). cDNA was synthesized from total cellular RNA using a RevertAid First Strand cDNA Synthesis Kit (Thermo Fisher Scientific, San Jose, CA, USA). qRT-PCR was performed using an ABI PRISM 7500 System (Applied Biosystems, Foster City, CA, USA; Life Technologies) and QuantiNova SYBR Green PCR Kit (Qiagen, Dusseldorf, Germany). The mRNA levels of CaM, CaMKK, CaMKIV, tau, and $\beta$-actin in hippocampal tissues and neurons were measured using qRT-PCR. The expression levels of each gene were calculated using the $2^{-\Delta \Delta C T}$ method [17]. The mRNA expression levels were displayed as fold changes compared with the internal control, $\beta$-actin. The primer sequences are shown in Table 1. 


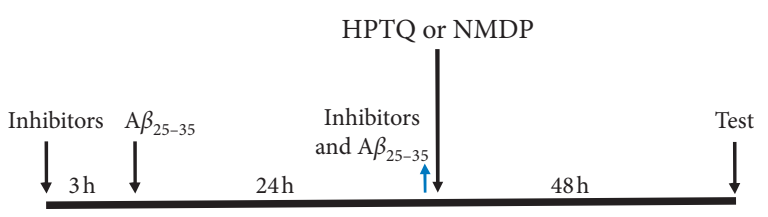

FIgURE 1: The time axis of drug intervention in hippocampal neurons. ৯: added; $\uparrow$ : removed.

2.6.2. Western Blot Analysis. The total cellular protein was extracted from hippocampal tissue or treated cells using a protein extraction kit, and the concentrations were measured using a Bicinchoninic Acid Kit (Beyotime Biotechnology, Shanghai, China). Equal amounts of protein were separated by $10 \%-12 \%$ SDS-PAGE (Bio-Rad) and transferred onto polyvinylidene fluoride (PDVF) membranes (Millipore, Billerica, MA, USA). After subsequently blocking in $5 \%$ nonfat dry milk for $2 \mathrm{~h}$, the membranes were incubated overnight at $4^{\circ} \mathrm{C}$ with the relevant primary antibody, followed by incubation with secondary antibodies for $1.5 \mathrm{~h}$ at $37^{\circ} \mathrm{C}$. The primary rabbit anti-rat monoclonal antibodies against CaM, CaMKK, p-CaMKIV, and p-tau were all obtained from Abcam (1:1000, Cambridge, UK). The secondary antibody, HRP-conjugated goat anti-rabbit IgG, was obtained from AmyJet Scientific Inc. (1:20,000, Abbkine, Wuhan, China). Rabbit anti-rat $\beta$-actin $(1: 2000$, Abbkine, AmyJet Scientific Inc.) was used as an internal control. The protein bands were visualized using electrogenerated chemiluminescence (ECL; Thermo Fisher Scientific) and analyzed on a gel imager (FluorChem M, ProteinSimple, USA).

2.7. Statistical Analysis. The measured data are presented as mean \pm standard deviation (SD). Multiple group means were compared by one-way analysis of variance and the LSD method. All analyses were performed using SPSS version 23.0 software, with a significance level set at $P<0.05$.

\section{Results}

3.1. Qualitative Analysis of HPTQ. Stilbene glycoside, ferulic acid, ginsenoside Rg1, aloe-emodin, $\beta$-asarone, emodin, and chrysophanol in HPTQ were determined by UPLC (Figure 2).

\subsection{Protective Effect of HPTQ on Neuronal Damage and Memory Improvement in AD Model Rats}

3.2.1. Learning and Memory in AD Model Rats. The Morris water maze was performed to evaluate spatial reference memory. In the escape latency test, the time taken to reach the platform was significantly higher in the MODEL group rats than in the CTRL group rats $(P<0.01)$. However, compared with the MODEL group rats, the time taken to reach the platform was significantly lower in the HPTQ and NMDP group rats $(P<0.05$ and $P<0.01)$. When comparing the number of times crossing the original platform, MODEL group rats had significantly lower frequencies
TABLE 1: Gene primer sequences of qRT-PCR.

\begin{tabular}{lcc}
\hline Primer & Sequence $\left(5^{\prime} \longrightarrow 3^{\prime}\right)$ & Length $(b p)$ \\
\hline CaM-F & CGACTTCCCTGAATTCCTGA & 20 \\
CaM-R & TCTGCTGCACTGATGTAGCC & 20 \\
CaMKK-F & CTTCAAGACCCACACCAGT & 19 \\
CaMKK-R & TGTAGAGTAAGGCCCAACC & 19 \\
CaMKIV-F & CCACATGGATACCGCTCAGA & 20 \\
CaMKIV-R & TGTTGGTGTGACTGCTGCTG & 20 \\
Tau-F & TCCACTGAGAACCTGAAGCA & 20 \\
Tau-R & TGTCCTTTGAGCCACACTTG & 20 \\
ACTIN-F & CCCGCGAGTACAACCTTCTTG & 21 \\
ACTIN-R & GTCATCCATGGCGAACTGGTG & 21 \\
\hline
\end{tabular}

than the CTRL group rats $(P<0.05$ or $P<0.01)$. Furthermore, compared with the MODEL group rats, the frequencies were significantly higher in the HPTQ and NMDP group rats (Figure 3 ).

\subsubsection{Effect of HPTQ on Hippocampal CA3 Region Ultra-} structure in AD Rats. The TEM results revealed that, in the CTRL group, tissue characteristics included intact nuclear membranes and condensed chromatin, with abundant organelles in the cytoplasm; in particular, a large number of ribosomes and rough endoplasmic reticulum were clearly visible. The cristae of mitochondria were intact, normal in structure, and without damage. In the MODEL group, the structure of neuronal nuclear membranes was blurred and irregular, and there were cytoplasmic edema and severe organelle damage. The mitochondria were swollen with some broken cristae, and vacuolar degeneration was apparent. In addition, most rough endoplasmic reticula were dilated, empty gun state, and even partly disappeared, with greatly reduced numbers of ribosomes. In the HPTQ and NMDP groups, nuclear membranes were relatively intact, and there were more ribosomes in the rough endoplasmic reticulum in the cytoplasm of neurons. A small number of mitochondria were slightly swollen and their cristae processes were destroyed (Figure 4).

3.3. Effect of HPTQ on Fluorescent p-Tau Expression in $A D$ Rats. Immunofluorescence was used to detect the fluorescent protein expression of $\mathrm{p}$-tau in the hippocampal CA3 region of $\mathrm{AD}$ rats. Compared with the CTRL group, the fluorescent expression of p-tau was significantly higher in the MODEL group, although the number of neurons was lower. However, compared with the MODEL group, the fluorescent expression of p-tau in the HPTQ and NMDP groups was significantly lower, while the number of neurons was higher (Figure 5).

3.4. Protective Effect of HPTQ-Medicated Serum on A $\beta_{25-35^{-}}$ Induced Neurotoxicity in Rat Hippocampal Neurons. To further determine the protective effect of HPTQ-medicated serum in rat hippocampal neurons, cell viability and cytotoxicity were measured using MTT. After the establishment of a cell model induced by $\mathrm{A} \beta_{25-35}$, either $5 \%$, 


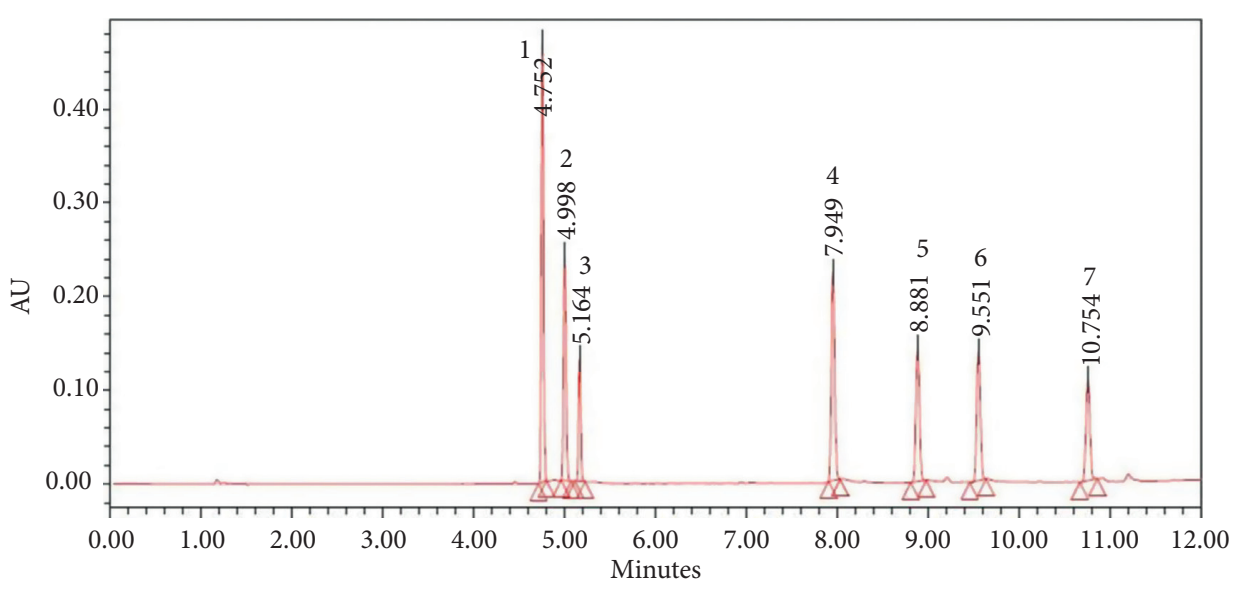

(a)

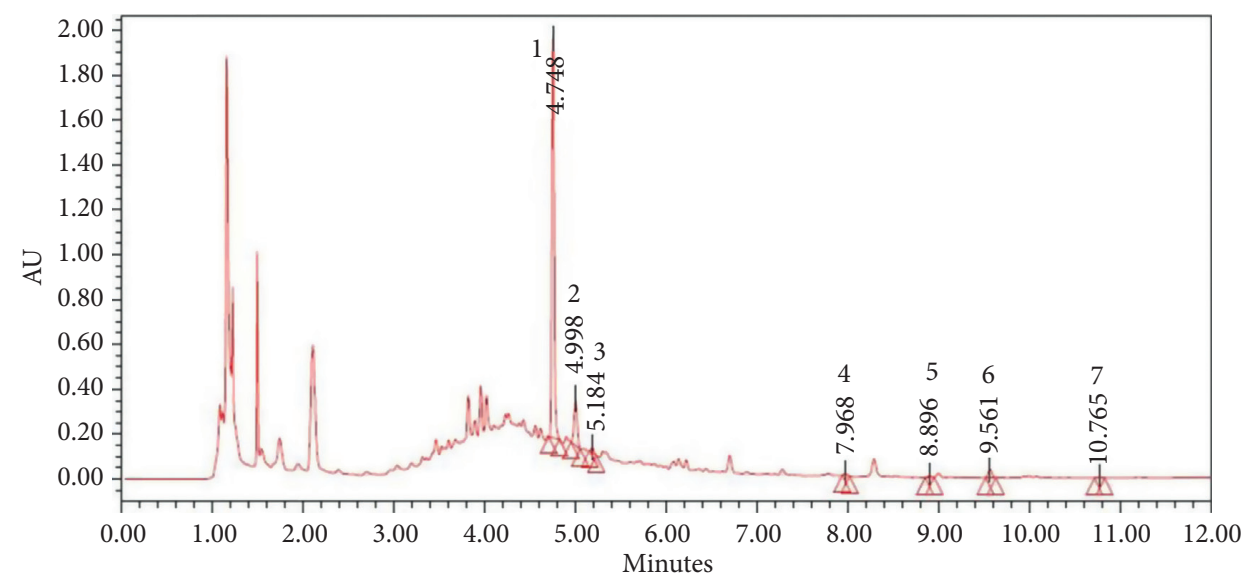

(b)

Figure 2: Peak chromatograms of Huang-Pu-Tong-Qiao (HPTQ). Mixed standard chromatogram: (1) stilbene glycoside; (2) ferulic acid; (3) ginsenoside Rg1; (4) aloe-emodin; (5) $\beta$-asarone; (6) emodin; (7) chrysophanol (a) and HPTQ chromatogram (b).

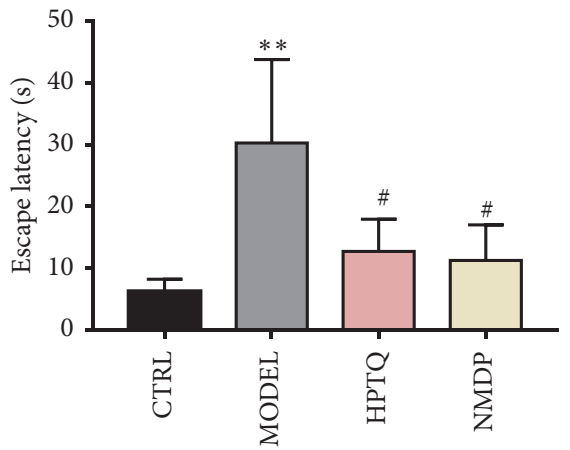

(a)

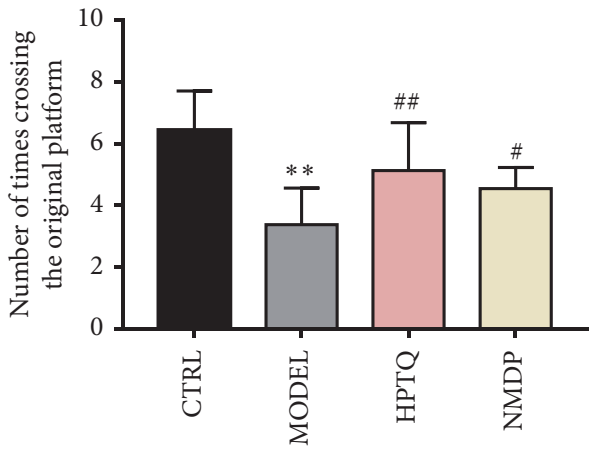

(b)

FIGURE 3: Effect of HPTQ on learning and memory in AD model rats. Effect of HPTQ on escape latencies in AD model rats (a). The number of times of crossing the original platform location in $\mathrm{AD}$ model rats (b). Values are expressed as the mean $\pm \mathrm{SD}$. ${ }^{*} P<0.05$, ${ }^{* *} P<0.01$, vs. CTRL group; ${ }^{\#} P<0.05,{ }^{\# \#} P<0.01$, vs. MODEL group.

$10 \%$, or $15 \%$ HPTQ-medicated serum and nimodipine solution was continued to intervene for another $24 \mathrm{~h}$. Compared with the CTRL group, the cell survival rate was significantly lower in the MODEL group $(P<0.01)$.
However, compared with the MODEL group, the cell survival rate was significantly higher in the $10 \%$ and $15 \%$ HPTQ-medicated serum groups and the NMDP group $(P<0.05$ or $P<0.01$; Figure 6$)$. 


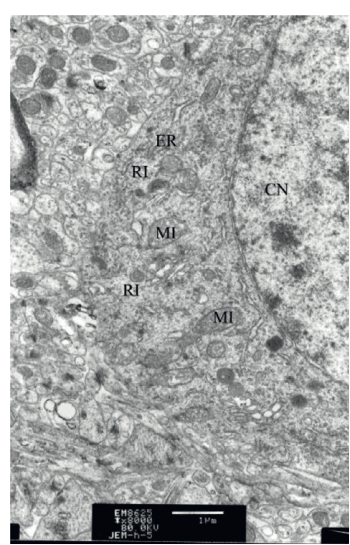

(a)

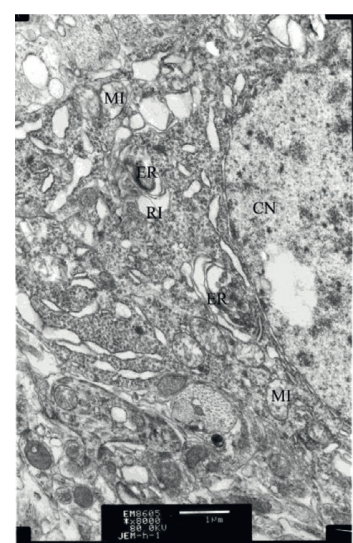

(b)

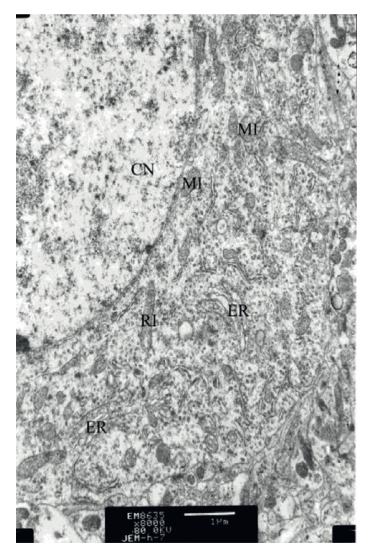

(c)

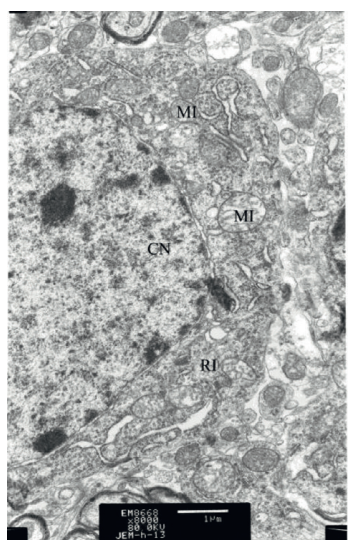

(d)

FIGURE 4: Effect of HPTQ against A 325 -35-induced neuronal impairment in AD rats. CTRL group (a). MODEL group (b). HPTQ group (c). NMDP group (d). CN: nucleus; RI: ribosome; MI: mitochondria; ER: endoplasmic reticulum.
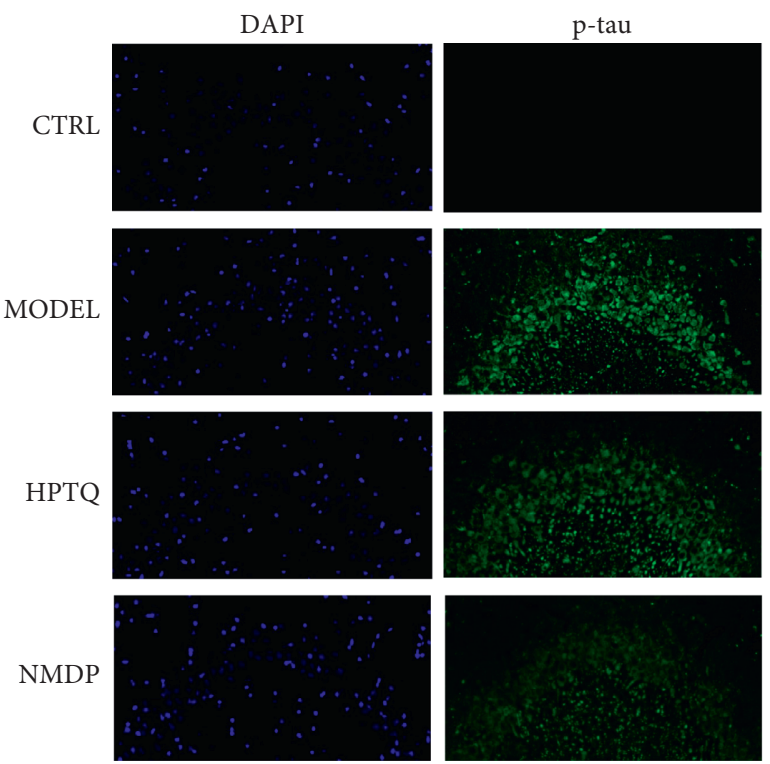

(a)
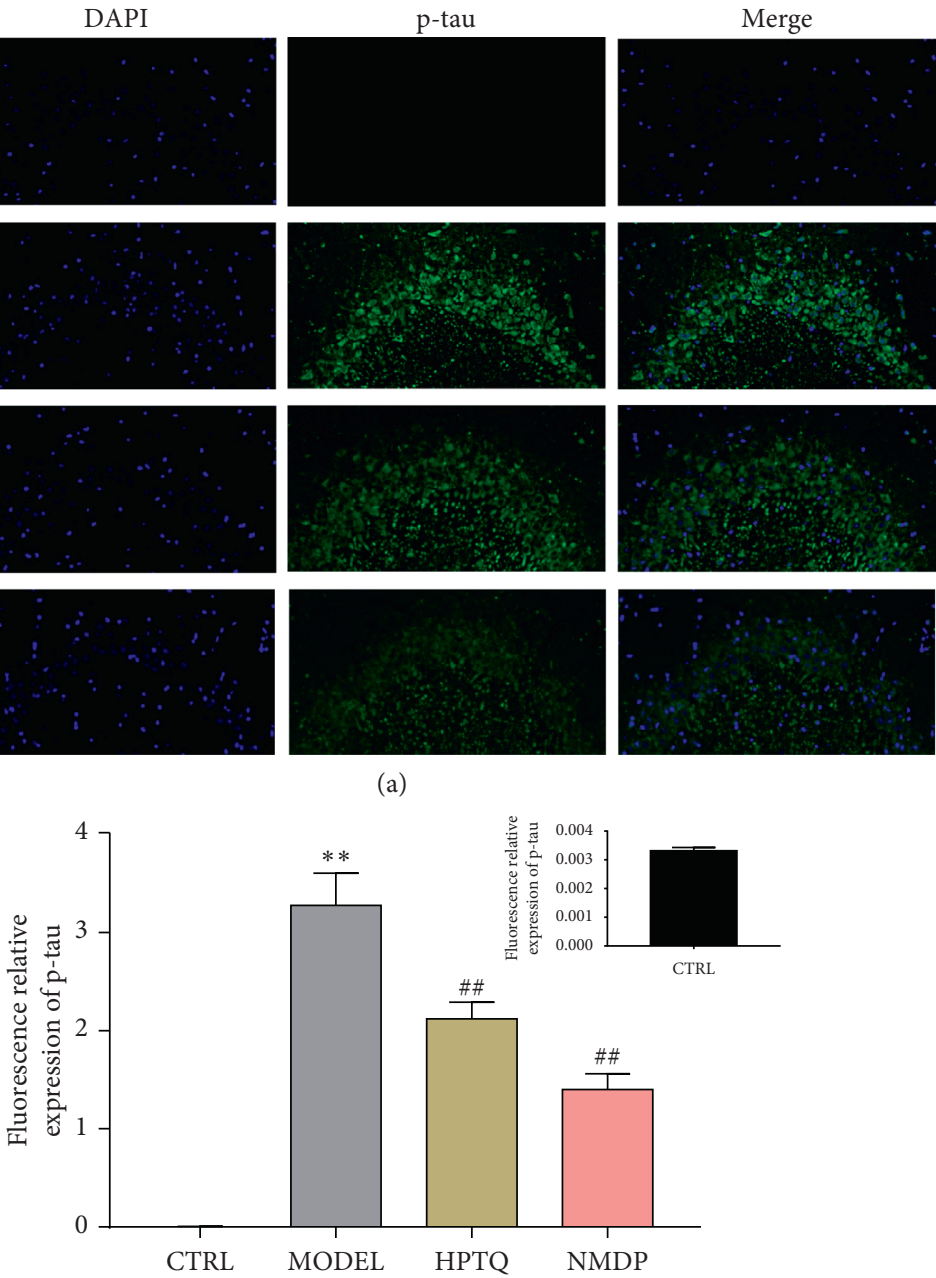

(b)

FIGURE 5: Effect of HPTQ on fluorescent protein expression of p-tau in AD rats. Immunofluorescence microscopic images of the CA3 region of the hippocampus (a). p-Tau ${ }^{+}$area/DAPI ${ }^{+}$area (b). Values are expressed as the mean $\pm \mathrm{SD} .{ }^{*} P<0.05,{ }^{* *} P<0.01$, vs. CTRL group; ${ }^{\#} P<0.05,{ }^{\#} P<0.01$, vs. MODEL group. 


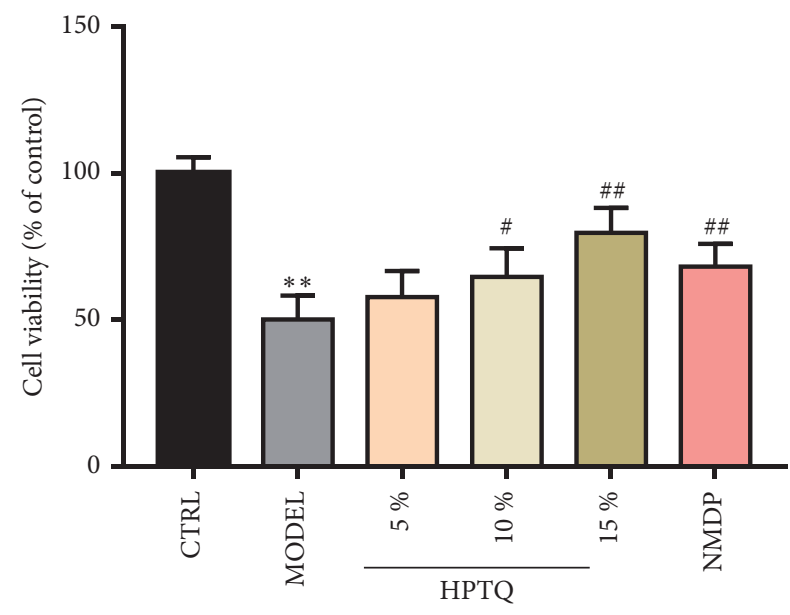

Figure 6: Protective effect of Huang-Pu-Tong-Qiao (HPTQ)medicated serum on the cell survival rate induced by $\mathrm{A} \beta_{25-35}$. Values are expressed as the mean \pm SD. ${ }^{*} P<0.05,{ }^{* *} P<0.01$, vs. CTRL group; ${ }^{\#} P<0.05,{ }^{\#} P<0.01$, vs. MODEL group.

3.5. Effect of HPTQ on Relevant mRNA Levels in the CaMCaMKIV Pathway in AD In Vivo and In Vitro Models. The mRNA expressions of CaM, CaMKK, CaMKIV, and tau were significantly higher in the MODEL group tissue compared with the CTRL group $(P<0.01)$. Compared with the MODEL group, the mRNA expressions of CaM, CaMKK, CaMKIV, and tau in the HPTQ and NMDP groups were significantly lower $(P<0.01$; Figure 7$)$. To further identify the role of the CaM-CaMKIV pathway in tau hyperphosphorylation, we used KN-62, a CaMKIV inhibitor [18], either separately or as a cotreatment with HPTQ in a cell culture model. Compared with the CTRL group, the mRNA levels of CaM, CaMKK, CaMKIV, and tau were significantly higher in the MODEL group $(P<0.01)$. Compared with the MODEL group, the mRNA expressions of CaM and CaMKK in the KN62 group were not statistically different, while the expression levels of CaMKIV and tau mRNA were significantly lower $(P<0.01)$. Furthermore, compared with the MODEL group, the mRNA levels of CaM, CaMKK, CaMKIV, and tau in the HPTQ, KN62 + HPTQ, and NMDP groups were significantly lower $(P<0.01$; Figure 8$)$.

3.6. Effect of HPTQ on Relevant Protein Expression Levels in the CaM-CaMKIV Pathway in AD In Vivo and In Vitro Models. In rat tissue, the protein expressions of CaM, CaMKK, p-CaMKIV, and p-tau were significantly higher in the MODEL group than in the CTRL group $(P<0.01)$. Compared with the MODEL group, the protein expressions of CaM, CaMKK, p-CaMKIV, and p-tau in HPTQ and NMDP group were significantly lower $(P<0.01$; Figure 9$)$. After separate or cotreatment with KN-62 in a cell culture model, compared with the CTRL group, the protein expressions of CaM, CaMKK, $\mathrm{p}$-CaMKIV, and $\mathrm{p}$-tau protein in the MODEL group were significantly higher $(P<0.01)$. Compared with the MODEL group, the protein expressions of CaM and CaMKK in the KN62 group were not statistically different, while the protein expressions of CaM and CaMKK in the HPTQ, KN62+HPTQ, and NMDP groups were significantly lower $(P<0.01)$. Compared with the MODEL group, the protein expressions of $\mathrm{p}$-CaMKIV and p-tau were significantly lower in the KN62, HPTQ, KN62+HPTQ, and NMDP groups $(P<0.01$; Figure 10).

\section{Discussion}

In the present study, we investigated the neuroprotective effect of HPTQ against $\mathrm{A} \beta$-induced toxicity in rats and rat hippocampal neurons. We also examined the CaM-CaMKIV pathway mediation of tau phosphorylation. The results revealed that HPTQ improved hippocampal neuronal damage and increased cell viability. In addition, HPTQ decreased tau phosphorylation via inhibition of the CaMCaMKIV pathway. In addition, the use of KN-62, a CaMKIV inhibitor, further demonstrated that HPTQ decreases tau phosphorylation via inhibition of the CaM-CaMKIV pathway.

Extracellular senile plaques, intracellular NFTs, and neuronal loss are the characteristic pathologies of $\mathrm{AD}$, which lead to learning and memory impairments [19]. The main component of senile plaques is $\mathrm{A} \beta$, forming insoluble fibrillar deposits in AD patients [20], and these mediate neuronal degeneration and loss. A previous study demonstrated that an $\mathrm{A} \beta$ injection into the rodent hippocampus results in physiological and cognitive deficits [21]. In addition, $\mathrm{A} \beta$ is extensively used to induce in vivo and in vitro models of $\mathrm{AD}$ because it is a neurotoxin [22]. $\mathrm{A} \beta_{25-35}$ forms $\beta$-sheet structures and amyloid-like fibrils and is likely endogenously produced by the enzymatic cleavage of $\mathrm{A} \beta_{1-40}$ when injected into the lateral ventricle [23]. In the present study, we used an intraperitoneal injection of $100 \mathrm{mg} / \mathrm{kg} \mathrm{D}$-galactose, combined with a lateral ventricle injection of $2 \mathrm{mg} / \mathrm{mL} \mathrm{A} \beta_{25-35}$, to establish an AD rat model. We also used $30 \mu \mathrm{M} \mathrm{A} \beta_{25-35}$ to induce the establishment of an $\mathrm{AD}$ cell model. We then demonstrated that HPTQ promoted learning and memory in $\mathrm{AD}$ rats and increased cell viability.

We also specifically examined the CaM-CaMKIV pathway-mediated reduction of tau phosphorylation. In $\mathrm{AD}$, there is a wide imbalance in $\mathrm{Ca}^{2+}$ signaling homeostasis [24]. Previous research has shown that $\mathrm{A} \beta$ enhances $\mathrm{Ca}^{2+}$ entry and that its overload aggravates $\mathrm{Ca}^{2+}$ imbalance [25]. CaM is a cytosolic protein that is expressed in all eukaryotic cells. It is the main receptor for $\mathrm{Ca}^{2+}$ in cells and combines with $\mathrm{Ca}^{2+}$ to form a $\mathrm{Ca}^{2+}$ / CaM complex that participates in modulating cellular signaling pathways [26]. CaMK is one kind of signaling protein that is regulated by $\mathrm{Ca}^{2+} / \mathrm{CaM}$. Multifunctional CaMKs are abundant in the brain and are activated by combining with $\mathrm{Ca}^{2+} / \mathrm{CaM}$, such as members of the CaMK cascade (CaMKK and CaMKIV) that alter and regulate the functionality of numerous protein substrates by phosphorylating them. For example, CaMKIV can be activated by CaMKK phosphorylating the kinase in its 


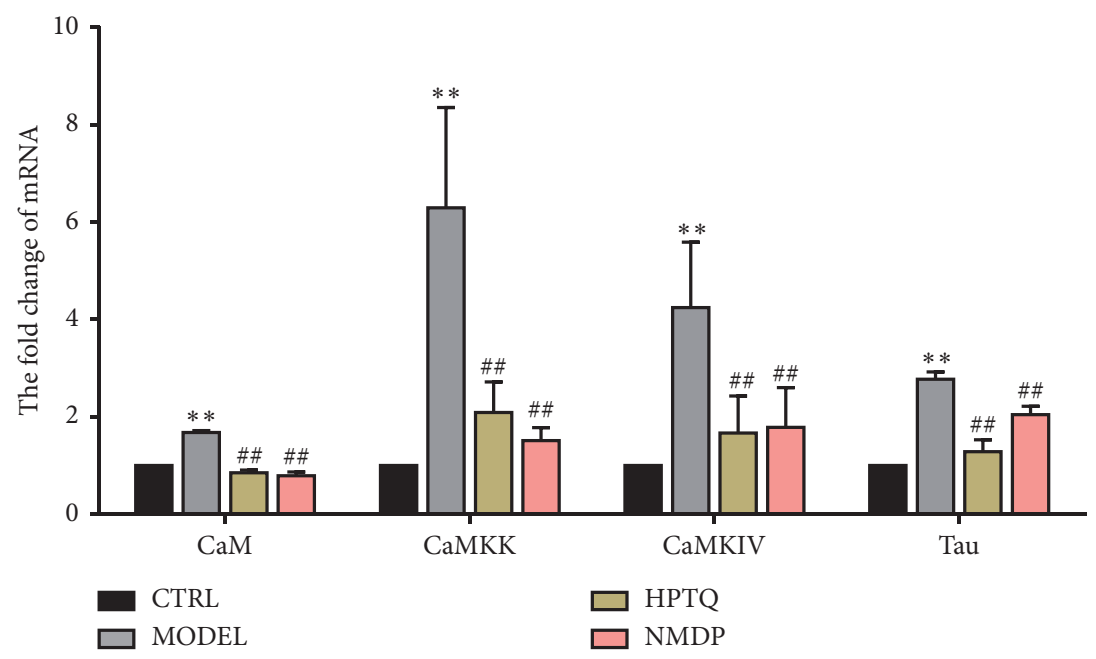

Figure 7: Effect of Huang-Pu-Tong-Qiao (HPTQ) on mRNA levels of CaM, CaMKK, CaMKIV, and tau in AD model rats. Values are expressed as the mean \pm SD $(n=3) .{ }^{*} P<0.05,{ }^{* *} P<0.01$, vs. CTRL group; ${ }^{\#} P<0.05,{ }^{\#} P<0.01$, vs. MODEL group.

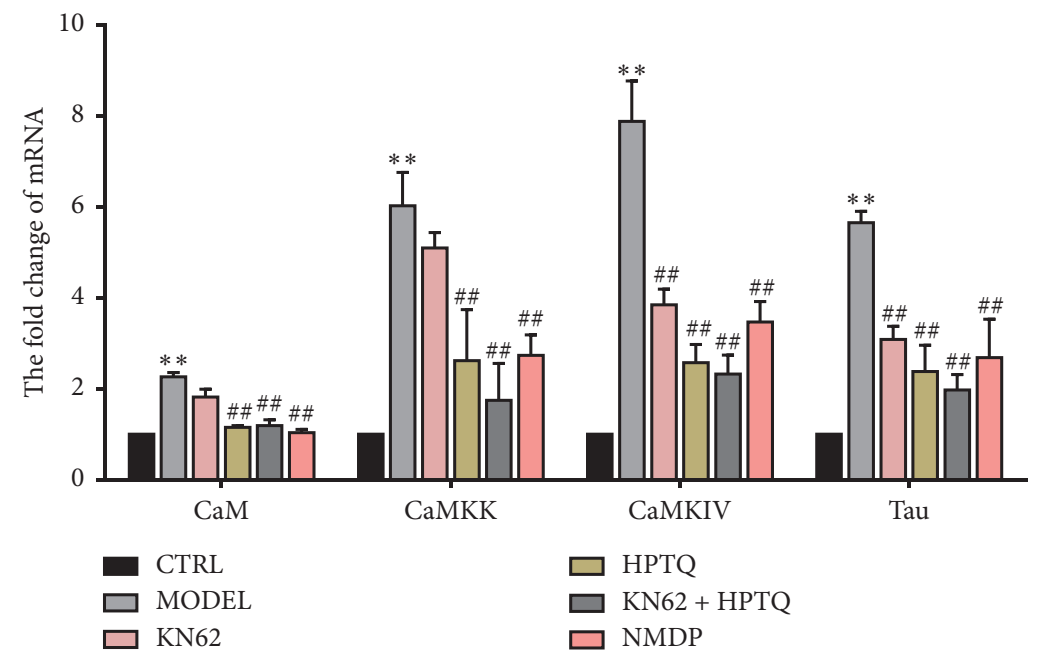

Figure 8: Effect of Huang-Pu-Tong-Qiao- (HPTQ-) medicated serum on mRNA levels of CaM, CaMKK, CaMKIV, and tau in primary hippocampal neurons. Values are expressed as the mean $\pm \mathrm{SD}(n=3) .{ }^{*} P<0.05,{ }^{* *} P<0.01$, vs. CTRL group; ${ }^{\#} P<0.05$, ${ }^{\# \#} P<0.01$, vs. MODEL group.

activation loop on Thr196 [27]. CaMKIV has a two-way regulatory function and can either promote or inhibit cell proliferation by regulating transcription factors. The multifunctional CaMKIV kinase has multiple substrates for activation, including tau.

Tau hyperphosphorylation can lead to the formation of NFTs in the AD brain, and pathological tau-induced neurotoxicity therefore plays a key role in driving neurodegenerative dysfunction [28]. Some studies have reported that overactivation of kinases and inactivation of phosphatases can lead to tau hyperphosphorylation [29], such as glycogen synthase kinase-3 $\beta$ (GSK-3 $\beta$ ), Janus kinase (JNK), mitogen-activated protein kinase (MAPK), and $\mathrm{Ca}^{2+} /$ calmodulin-dependent kinase
$[30,31]$. In previous studies, TianDiJingWan treatment significantly reduced p-tau aggregation and improved learning and memory in an $\mathrm{AD}$ rat model [32], and the $\mathrm{A} \beta$-N-terminus monoclonal antibody (mAb) A8 decreased levels of both $\mathrm{A} \beta$ and $\mathrm{p}$-tau in the brains of APP/ PS1 mice and alleviated cognitive dysfunction [33]. Therefore, the inhibition of tau hyperphosphorylation can meaningfully improve both neuronal damage and cognitive function.

The HPTQ used in the current study was mainly composed of Radix Rhei Et Rhizome, Chuanxiong Rhizoma, Panax Ginseng C. A. Mey, Acori Tatarinowii Rhizoma, Fructus Alpinae Oxyphyllae, and Radix Polygoni Multiflori Praeparata. Modern pharmacological 


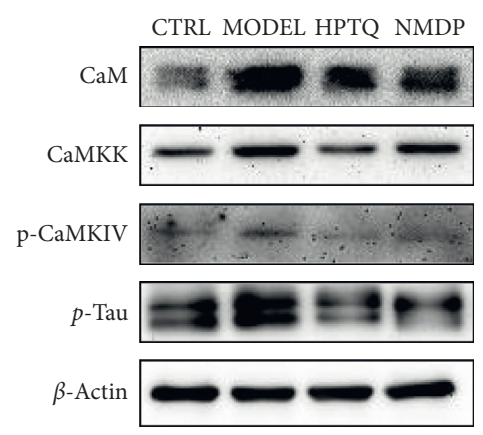

(a)

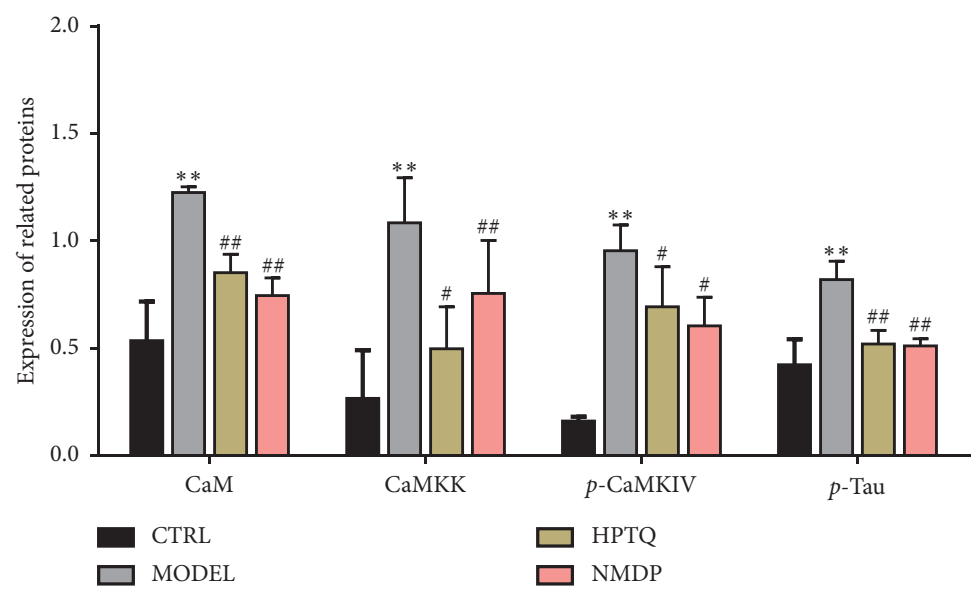

(b)

FIGURe 9: Effect of HPTQ on the protein expressions of CaM, CaMKK, p-CaMKIV, and p-tau in the in vivo AD model. The gray bands of different proteins (a). The graph displays densitometric analyses of the expression ratios of CaM, CaMKK, p-CaMKIV, and p-tau (b). Values are expressed as the mean \pm SD $(n=3) .{ }^{*} P<0.05,{ }^{* *} P<0.01$, vs. CTRL group; ${ }^{\#} P<0.05,{ }^{\# \#} P<0.01$, vs. MODEL group.

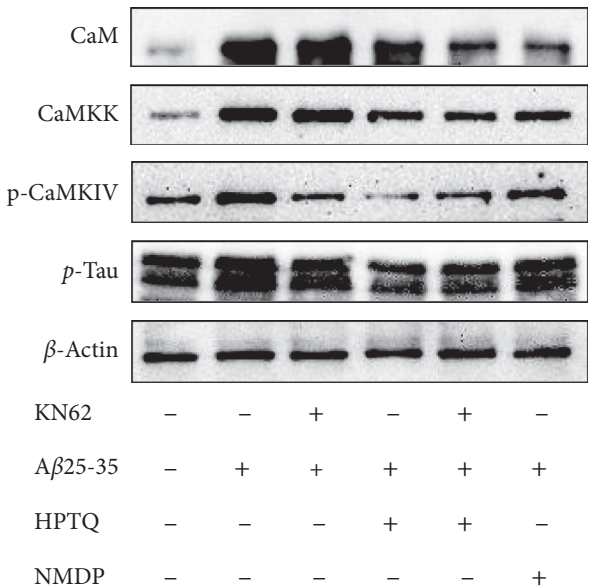

(a)

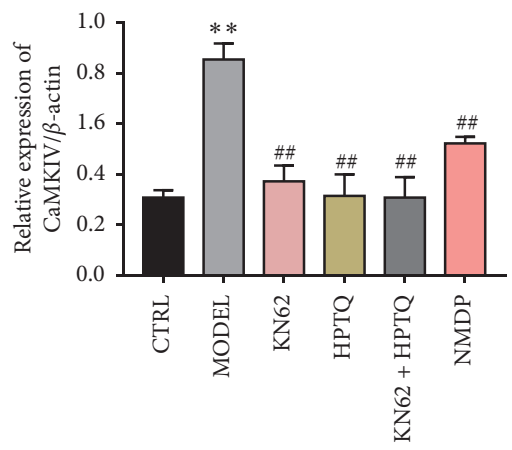

(d)
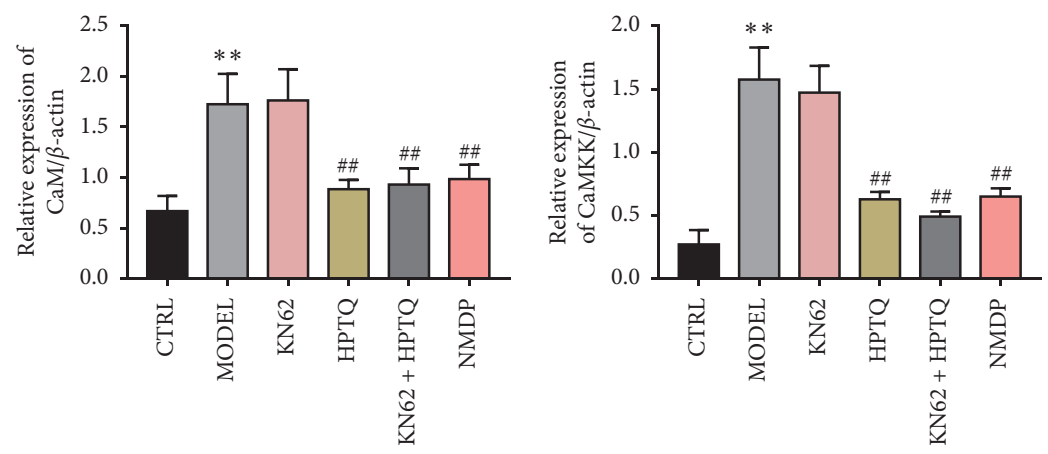

(c)

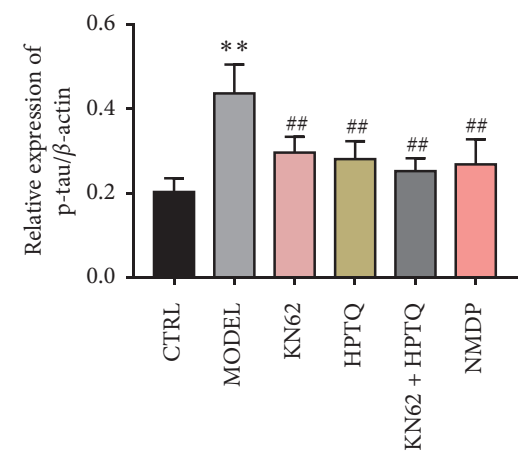

(e)

FIGURE 10: Effect of HPTQ on the protein expressions of CaM, CaMKK, p-CaMKIV, and p-tau in the in vitro AD model. The gray bands of different proteins (a). The graph displays densitometric analyses of the expression ratios of CaM, CaMKK, p-CaMKIV, and p-tau (b). Values are expressed as the mean $\pm \mathrm{SD}(n=3) .{ }^{*} P<0.05,{ }^{* *} P<0.01$, vs. CTRL group; ${ }^{\#} P<0.05,{ }^{\# \#} P<0.01$, vs. MODEL group.

studies have indicated that ginseng is an effective drug for protecting the nervous system and that it can improve and relieve stress and fatigue and prevent neurodegenerative diseases [34]. Ginsenoside Rg1, an active ingredient of ginseng, was reported to improve cognitive impairment and memory in SAMP8 mice and may regulate PKA/ CREB activity via the blood-brain barrier [35]; the effective ingredients of rhubarb are anthraquinone 
derivatives, including emodin, aloe-emodin, chrysophanol, rhein, physcion, and danthron, and these can improve central nervous system diseases such as traumatic brain injury, brain tumors, and Alzheimer's disease [36]. Furthermore, $\beta$-asarone can improve cognitive function by suppressing neuronal apoptosis in an $\mathrm{AD}$ rat model induced by A $\beta$ [37], and combined with tenuigenin, it can significantly improve the efficacy of memantine in treating moderate-to-severe $\mathrm{AD}$ [38]. Tetrahydroxystilbene glucoside (TSG) is the major active component of Polygonum multiflorum, which can improve cognitive deficits in AD rats [39]. Ferulic acid, an antioxidant, is the main component of Ligusticum chuanxiong, which can protect the brain from $\mathrm{A} \beta$ neurotoxicity and neuronal death caused by ROS [40].

Results from our study suggest that the $\mathrm{A} \beta$ toxin activates the CaM-CaMKIV pathway, leading to tau hyperphosphorylation, whereas HPTQ and the positive control NMDP attenuated this effect. Moreover, treatment with KN-62 only, as well as cotreatment with HPTQ and KN-62, significantly inhibited tau phosphorylation. However, there were no statistically significant differences between treatment with HPTQ only and cotreatment with HPTQ and $\mathrm{KN}-62$. These results imply that HPTQ protects against hippocampal neuronal damage and tau phosphorylation induced by $\mathrm{A} \beta_{25-35}$ and improves learning and memory in $\mathrm{AD}$ rats, and suggest that these effects may occur via inhibition of the CaM-CaMKIV pathway.

\section{Conclusions}

For more than 10 years, HPTQ has been used as an inhospital preparation for the clinical treatment of dementia. However, the mechanisms of its effect are unclear. Our results show that:

(1) HPTQ is a multiflavored Chinese medicine composition that contains stilbene glycoside, ferulic acid, ginsenoside Rg1, aloe-emodin, $\beta$-asarone, emodin, and chrysophanol as its various chemical components

(2) HPTQ is protective against hippocampal neuronal damage and reduces hippocampal neuronal loss and acts as a neuroprotective agent in an $\mathrm{AD}$ rat model

(3) HPTQ reduces tau phosphorylation via inhibition of the CaM-CaMKIV pathway

Overall, our study revealed a potential mechanism of HPTQ in the treatment of $\mathrm{AD}$, thus providing new ideas for the development of therapeutic AD drugs based on eliminating the potentially pathological product tau.

\section{Data Availability}

The data used to support the findings of this study are available upon request by contact with the corresponding author.

\section{Conflicts of Interest}

The authors declare no conflicts of interest.

\section{Acknowledgments}

This work was supported by the National Natural Science Foundation of China (grants nos. 81574040 and 81873351), Distinguished Young Scholars Project of Natural Science Foundation of Anhui Province in China (grant no. 1908085J27), and General Project Foundation of Support Program for the Excellent Young Faculties in Universities of Anhui Province in China (grant no. gxyq2019033).

\section{References}

[1] A. Kumar, A. Singh, and Ekavali, "A review on Alzheimer's disease pathophysiology and its management: an update," Pharmacological Reports, vol. 67, no. 2, pp. 195-203, 2015.

[2] R. Anand, K. D. Gill, and A. A. Mahdi, "Therapeutics of Alzheimer's disease: past, present and future," Neuropharmacology, vol. 76, pp. 27-50, 2014.

[3] B. De Strooper and E. Karran, "The cellular phase of Alzheimer's disease," Cell, vol. 164, no. 4, pp. 603-615, 2016.

[4] A. Wimo, M. Guerchet, G.-C. Ali et al., "The worldwide costs of dementia 2015 and comparisons with 2010," Alzheimer's \& Dementia, vol. 13, no. 1, pp. 1-7, 2017.

[5] P. Sharma, P. Srivastava, A. Seth, P. N. Tripathi, A. G. Banerjee, and S. K. Shrivastava, "Comprehensive review of mechanisms of pathogenesis involved in Alzheimer's disease and potential therapeutic strategies," Progress in Neurobiology, vol. 174, pp. 53-89, 2019.

[6] H. Kadavath, R. V. Hofele, J. Biernat et al., "Tau stabilizes microtubules by binding at the interface between tubulin heterodimers," in Proceedings of the National Academy of Sciences, vol. 112, no. 24, pp. 7501-7506, Washington, CO, USA, June 2015.

[7] W. Noble, D. P. Hanger, C. C. Miller et al., "The importance of tau phosphorylation for neurodegenerative diseases," Frontiers in Neurology, vol. 4, p. 83, 2013.

[8] J. Biernat, N. Gustke, G. Drewes, E. Mandelkow, and E. Mandelkow, "Phosphorylation of Ser262 strongly reduces binding of tau to microtubules: distinction between PHF-like immunoreactivity and microtubule binding," Neuron, vol. 11, no. 1, pp. 153-163, 1993.

[9] L. M. Ittner and J. Gotz, "Amyloid-beta and tau-a toxic pas de deux in Alzheimer's disease," Nature Reviews Neuroscience, vol. 12, no. 2, pp. 65-72, 2011.

[10] G. Gallardo and D. M. Holtzman, "Antibody therapeutics targeting abeta and tau," Cold Spring Harbor Perspectives in Medicine, vol. 7, no. 10, 2017.

[11] L. Serenó, M. Coma, M. Rodríguez et al., “A novel GSK-3 $\beta$ inhibitor reduces Alzheimer's pathology and rescues neuronal loss in vivo," Neurobiology of Disease, vol. 35, no. 3, pp. 359-367, 2009.

[12] J. M. Long and D. M. Holtzman, "Alzheimer disease: an update on pathobiology and treatment strategies," Cell, vol. 179, no. 2, pp. 312-339, 2019.

[13] G. Paxinos and C. Watson, "The Rat Brain in Stereotic Coordinates," Academic Press Inc., vol. 191, San Diego, CA, USA, 3rd edition, 1997.

[14] C. V. Vorhees and M. T. Williams, "Morris water maze: procedures for assessing spatial and related forms of learning and memory," Nature Protocols, vol. 1, no. 2, pp. 848-858, 2006.

[15] E. M. Korkotian and M. Segal, "Calcium-containing organelles display unique reactivity to chemical stimulation in 
cultured hippocampal neurons," The Journal of Neuroscience, vol. 17, no. 5, pp. 1670-1682, 1997.

[16] M. A. Kuszczyk, S. Sanchez, J. Pankiewicz et al., "Blocking the interaction between apolipoprotein $\mathrm{E}$ and $\mathrm{A} \beta$ reduces intraneuronal accumulation of $\mathrm{A} \beta$ and inhibits synaptic degeneration," The American Journal of Pathology, vol. 182, no. 5, pp. 1750-1768, 2013.

[17] L. E. Jarrard, "On the role of the hippocampus in learning and memory in the rat," Behavioral and Neural Biology, vol. 60, no. 1, pp. 9-26, 1993.

[18] T. Kato, M. Sano, S. Miyoshi et al., "Calmodulin kinases II and IV and calcineurin are involved in leukemia inhibitory factorinduced cardiac hypertrophy in rats," Circulation Research, vol. 87 , no. 10 , pp. 937-945, 2000.

[19] D. J. Selkoe, "Translating cell biology into therapeutic advances in Alzheimer's disease," Nature, vol. 399, no. 6738, pp. A23-A31, 1999.

[20] D. J. Selkoe, "Alzheimer's disease: genes, proteins, and therapy,” Physiological Reviews, vol. 81, no. 2, pp. 741-766, 2001.

[21] C. Zussy, A. Brureau, B. Delair et al., "Time-course and regional analyses of the physiopathological changes induced after cerebral injection of an amyloid $\beta$ fragment in rats," The American Journal of Pathology, vol. 179, no. 1, pp. 315-334, 2011.

[22] C. Tohda, T. Tamura, S. Matsuyama, and K. Komatsu, "Promotion of axonal maturation and prevention of memory loss in mice by extracts ofAstragalus mongholicus," British Journal of Pharmacology, vol. 149, no. 5, pp. 532-541, 2006.

[23] T. Kubo, S. Nishimura, Y. Kumagae, and I. Kaneko, "In vivo conversion of racemized beta-amyloid ([D-Ser26] A beta $1-40)$ to truncated and toxic fragments ([D-Ser26]A beta 25-35/40) and fragment presence in the brains of Alzheimer's patients," Journal of Neuroscience Research, vol. 70, no. 3, pp. 474-483, 2002.

[24] N. Pierrot, S. F. Santos, C. Feyt, M. Morel, J.-P. Brion, and J.-N. Octave, "Calcium-mediated transient phosphorylation of tau and amyloid precursor protein followed by intraneuronal amyloid- $\beta$ accumulation," Journal of Biological Chemistry, vol. 281, no. 52, pp. 39907-39914, 2006.

[25] K. V. Kuchibhotla, S. T. Goldman, C. R. Lattarulo, H.-Y. Wu, B. T. Hyman, and B. J. Bacskai, "A $\beta$ plaques Lead to aberrant regulation of calcium homeostasis in vivo resulting in structural and functional disruption of neuronal networks," Neuron, vol. 59, no. 2, pp. 214-225, 2008.

[26] D. Chin and A. R. Means, "Calmodulin: a prototypical calcium sensor," Trends in Cell Biology, vol. 10, no. 8, pp. 322-328, 2000.

[27] M. T. Swulius and M. N. Waxham, " $\mathrm{Ca}^{2+} /$ Calmodulin-dependent protein kinases," Cellular and Molecular Life Sciences, vol. 65, no. 17, pp. 2637-2657, 2008.

[28] P. Friedhoff, A. Schneider, E.-M. Mandelkow, and E. Mandelkow, "Rapid assembly of Alzheimer-like paired helical filaments from microtubule-associated protein tau monitored by fluorescence in solution," Biochemistry, vol. 37, no. 28, pp. 10223-10230, 1998.

[29] Y. Shi, M. Manis, J. Long et al., "Microglia drive APOE-dependent neurodegeneration in a tauopathy mouse model," Journal of Experimental Medicine, vol. 216, no. 11, pp. 2546-2561, 2019.

[30] F. Hernández, E. Gómez de Barreda, A. Fuster-Matanzo, J. J. Lucas, and J. Avila, "GSK3: a possible link between beta amyloid peptide and tau protein," Experimental Neurology, vol. 223, no. 2, pp. 322-325, 2010.
[31] H. Coman, B. Nemeş, Nemeş et al., "New therapeutic targets in Alzheimer's disease," International Journal of Gerontology, vol. 11, no. 1, pp. 2-6, 2017.

[32] Z. Li, Q. Tong, H. Xu et al., "Therapeutic effects of TianDiJingWan on the abeta 25-35-induced Alzheimer's disease model rats," Evidence-Based Complementary and Alternative Medicine, vol. 2015, Article ID 307350, 9 pages, 2015.

[33] S. Xiao, L.-L. Song, J.-T. Li et al., "Intraperitoneal administration of monoclonal antibody against pathologic $\mathrm{A} \beta 42$ aggregates alleviated cognitive deficits and synaptic Lesions in APP/PS1 mice," Journal of Alzheimer's Disease, vol. 73, no. 2, pp. $657-670,2020$.

[34] S.-Y. Nah, "Ginseng ginsenoside pharmacology in the nervous system: involvement in the regulation of ion channels and receptors," Frontiers in Physiology, vol. 5, p. 98, 2014.

[35] Y.-Q. Shi, T.-W. Huang, L.-M. Chen et al., "Ginsenoside Rg1 attenuates amyloid- $\beta$ content, regulates PKA/CREB activity, and improves cognitive performance in SAMP8 mice," Journal of Alzheimer's Disease, vol. 19, no. 3, pp. 977-989, 2010.

[36] X. Li, S. Chu, Y. Liu et al., "Neuroprotective effects of anthraquinones from rhubarb in central nervous system diseases," Evidence Based Complementary Alternative Medicine, vol. 2019, Article ID 3790728, 12 pages, 2019.

[37] Y. Geng, C. Li, J. Liu et al., "Beta-asarone improves cognitive function by suppressing neuronal apoptosis in the beta-amyloid Hippocampus injection rats," Biological \& Pharmaceutical Bulletin, vol. 33, no. 5, pp. 836-843, 2010.

[38] B. L. Wang, L. Xuan, S. J. Dai et al., "[Protective effect of betaasarone on $\mathrm{AD}$ rat model induced by intracerebroventricular injection of Abeta (1)(-)(4)(2) combined 2-VO and its mechanism]," Zhongguo Zhong Yao Za Zhi, vol. 42, no. 24, pp. 4847-4854, 2017.

[39] L. Zhou, Y. Hou, Q. Yang et al., “Tetrahydroxystilbene glucoside improves the learning and memory of amyloid- $\beta 1-42$ injected rats and may be connected to synaptic changes in the hippocampus," Canadian Journal of Physiology and Pharmacology, vol. 90, no. 11, pp. 1446-1455, 2012.

[40] M. Benchekroun, A. Romero, J. Egea et al., "The antioxidant additive approach for Alzheimer's disease therapy: new ferulic (lipoic) acid plus melatonin modified tacrines as cholinesterases inhibitors, direct antioxidants, and nuclear factor (erythroid-derived 2)-like 2 activators," Journal of Medicinal Chemistry, vol. 59, no. 21, pp. 9967-9973, 2016. 